\title{
Estudio comparativo de diferentes trampas de luz (LEDs) con energía solar para la captura masiva de adultos polilla del tomate Tuta absoluta en invernaderos de tomate en la Provincia de Entre Ríos, Argentina
}

\author{
Comparative study among a variety of solar-powered LED traps \\ to capture tomato leafminers Tuta absoluta adults by mass trapping \\ in tomato greenhouses in the Province of Entre Ríos, Argentina
}

\author{
Jorge Castresana ${ }^{*}$, Laura Puhl 2
}

\begin{abstract}
RESUMEN
Existen a nivel mundial una gran variedad de artrópodos plaga que causan perjuicios en cultivos hortícolas. Entre estos se encuentra la polilla del tomate Tuta absoluta (Meyrick) (Lepidoptera: Gelechiidae) la cual ha sido considerada como una de las principales plagas que afectan al cultivo de tomate bajo cubierta. La polilla del tomate produce daño directo por medio de sus larvas que realizan galerías en las hojas, brotes y cáliz de frutos inmaduros, en su proceso alimenticio interfiriendo en la fotosíntesis y el aspecto estético del fruto lo cual provoca graves pérdidas económicas.

El trabajo se realizó con el objetivo de evaluar la efectividad de diferentes tipos de trampas caseras con diodos emisores de luces (LEDs) alimentadas con energía solar en combinación con feromona para la captura de adultos de polilla del tomate como alternativa ecológica de control en un cultivo protegido de tomate en Concordia, Argentina. Se probaron diferentes trampas: con luces LEDs 430, $470 \mathrm{~nm}$ solas o en combinación con feromona sexual, los cuales fueron comparados con una trampa testigo de feromona sexual. Para ello se realizó una un diseño en bloques completamente al azar con dos repeticiones (invernaderos). Las trampas fueron distribuidas en forma aleatoria y colocadas en los caminos a una distancia equidistante entre ellas parte las cuales fueron rotadas quincenalmente para no producir sesgos en las capturas. Se evaluó el número promedio de capturas totales (adultos de polilla de tomate). Los resultados mostraron que la trampa con LEDs de $470 \mathrm{~nm}$ combinada con feromona sexual registró un mayor número de capturas de polilla del tomate respecto al resto de las trampas.
\end{abstract}

Palabras clave: polilla del tomate, trampas, LEDs, captura masiva, tomate.

\section{ABSTRACT}

A wide variety of arthropod pests that cause damage in agricultural crops can be found worldwide. The tomato leafminer (Meyrick) (Lepidoptera: Gelechiidae) is considered to be one of the most significant pests which affects greenhouse tomato crops. The tomato leafminer causes direct damage when larvae produce galleries in leaves, shoots and calix of immature fruits throughout their feeding process, which affects the photosynthesis and makes the fruit unattractive resulting in serious economic loss. This research was carried out to investigate the efficacy of a variety of handmade mass trapping equipped with solar-powered Light Emitting Diodes (LEDS) and combined with pheromones, as an alternative biological control, in order to capture tomato leafminers adults in greenhouse tomato crops in Concordia, Argentina. Different types of traps were tested, namely, traps supplemented with LEDs 430, $470 \mathrm{~nm}$ with or without sex pheromones, which were compared to a witness trap of sex pheromone. The study was designed as a randomized complete block design replicated 2 times (greenhouses). These traps were placed at random and along the corridor at an even distance among one another changed on a two-weekly basis in order to eliminate bias from the capture results. These results showed that the total average number of tomato leafminer adults caught in the trap equipped with LEDs $470 \mathrm{~nm}$ in combination with sex pheromone was higher compared to the other traps.

Key words: tomato leafminer, traps, LEDs, massive trapping, tomato.

1 Estación Experimental Agropecuaria INTA Concordia, C.P. 3200, Estación Yuquerí, Concordia, Entre Ríos, Argentina.

2 Facultad de Agronomía, Cátedra de Modelos Cuantitativos Aplicados, Universidad de Buenos Aires, Buenos Aires, Argentina.

* Autor por correspondencia: castresana.jorge@correo.inta.gob.ar

Fecha de Recepción: 29 Junio, 2017.

Fecha de Aceptación: 20 Septiembre, 2017.

DOI: 


\section{Introducción}

Entre las principales plagas del cultivo de tomate Lycopersicon esculentum Mill. en América del Sur se encuentra Tuta absoluta (Meyrick) (Lepidoptera: Gelechiidae) (Pican ço et al., 2007). Este insecto es originario de América del Sur y se distribuye en un estrecho territorio limitado por Ecuador, Cordillera de los Andes, Norte de Chile y litoral del Océano Pacífico, incluyendo el archipiélago de las islas Galápagos (Giordano \& Silva, 1999). La polilla del tomate fue introducida en Europa vía España (2006) y, por su gran capacidad de dispersión, ha llegado prácticamente a todo el continente. Recientemente fue introducida en África y Asia, donde ha causado grandes perjuicios (Desneux et al., 2010). Está presente en los cultivos de tomate bajo cubierta en la Provincia de Entre Ríos, Argentina. Los daños causados por las larvas de este insecto afectan la productividad, ya que reducen el área fotosintética al alimentarse del mesófilo foliar (Bogorni et al., 2003) como así también afectan el crecimiento vertical al minar, tallo, brote apical, los botones florales, las flores y, principalmente, los frutos (Leite et al., 2004; Potting et al., 2009). Las pérdidas ocasionadas por este insecto varían entre los estadios fenológicos del tomate y a lo largo del año (Castelo Branco, 1992). En ataques severos, a causa de su alimentación, pueden destruir completamente las hojas del tomate, los brotes tiernos y comprometer los frutos en todas sus etapas (EPPO, 2011), preferentemente en los frutos inmaduros penetrando por el extremo del pedúnculo, lo cual favorece la entrada de patógenos que da como resultado un deterioro en su calidad para la comercialización (Potting et al., 2009). Es una plaga que exhibe un alto potencial de multiplicación, ya que los adultos son nocturnos y durante el día pueden encontrarse escondidos entre las hojas (Hariza nova, 2009). Si bien T. absoluta vuela a poca altura $(30-60 \mathrm{~cm})$, sobre la planta huésped (Al-Zaidi, 2009a), pueden desplazarse con gran facilidad varios kilómetros a la deriva con la ayuda del viento (van Deventer, 2009).

En Argentina esta plaga se controla exclusivamente a través del uso de insecticidas. La alta densidad de población de polilla del tomate en el cultivo requiere la aplicación reiterada de tratamientos con insecticidas de síntesis química (organofosforados y piretroides), cuyos resultados son tan poco eficaces
(Siquiera et al., 2000) y, tan importantes en un en cultivo bajo cubierta, que es necesario intensificar los tratamientos que inexorablemente conducen a un uso indiscriminado de estos productos de síntesis química. Ésto ocasiona los siguientes perjuicios: 1) destrucción del complejo de enemigos naturales, 2) incremento de costos de producción, 3) aumento del desarrollo de resistencia de poblaciones de la polilla a insecticidas; y 4) riesgos para la salud de productores, consumidores y contaminación ambiental.

En los cultivos de tomate bajo cubierta de la Provincia de Entre Ríos, el uso indiscriminado de insecticidas de síntesis química es el principal y casi exclusiva forma de control de la polilla del tomate. Entre los o dificultades que se presentan es, por una parte, que sólo algunos productores tienen acceso a utilizar insecticidas selectivos con principios activos para su control con bajo impacto, compatibles con la fauna auxiliar existente y, por otro lado, la falta de precaución en alternar el uso de las materias activas con diferentes formas de actuación, formulaciones y aplicaciones adecuadas.

La utilización de feromona sexual es una alternativa de control para la T. absoluta que reduce el uso de insecticidas. Esta feromona puede ser colocada en dos tipos de trampas. 1) trampa delta, con el propósito de realizar captura de adultos para hacer un seguimiento de la plaga. En este caso se puede determinar el riesgo para el cultivo (Cabello et al. 2010) teniendo en cuenta que no siempre el hecho de no capturar adultos se relaciona con la ausencia de la plaga, por lo tanto, debe monitorearse periódicamente el cultivo para detectar la presencia de orugas en folíolos. (Cabello et al. (2010), 2) trampa de agua, con la finalidad de realizar una captura masiva de adultos de polilla del tomate (Martí et al., 2010). Ambos tipos de trampas son complementos fundamentales en el marco del manejo integrado de plagas (MIP). Con respecto a la captura masiva, si bien existen varios estudios a campo realizados, éstos resultan tener una alta eficacia únicamente para la captura de machos temprano en la mañana (Ferrara et al., 2001). Considerando que cada hembra fecundada tiene una longevidad de días (24) y puede poner más de 250 huevos en los brotes como en el envés de las hojas, una pequeña proporción de éstas vivas podría generar graves daños al cultivo.

Sobre la premisa que los ojos compuestos de los insectos en general perciben el color por medio 


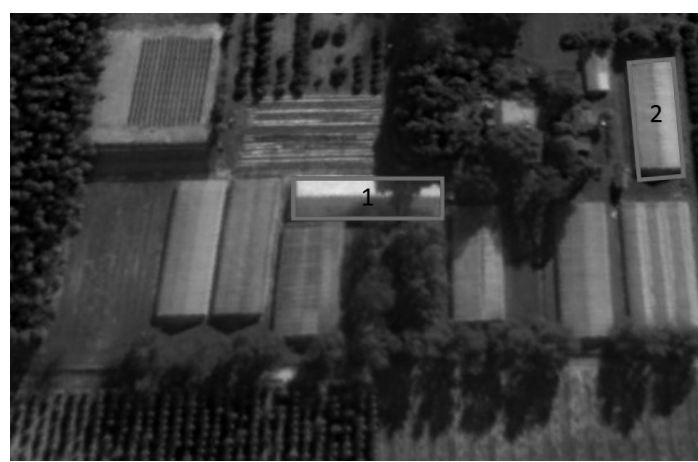

Figura 1. Vista aérea de la zona del ensayo en contorno verde, superficie del estudio.

de tres diferentes tipos de células fotorreceptores sensibles al espectro de la luz con picos en la región del ultravioleta, azul y verde, $(370-390 \mathrm{~nm}, 450$ - $470 \mathrm{~nm}$, y 530 - $550 \mathrm{~nm}$ ), respectivamente, los cuales se han conservado a través de su evolución. (Briscoe \& Chittka, 2001), se han llevado a cabo estudios en los cuales varias especies de polillas son atraídas por iluminación nocturna artificial, especialmente, con alta emisión de luz en la región del UV (Frank, 2006). Esta atracción está posiblemente determinada por la sensibilidad a la luz, lo cual se supone está relacionada con el tamaño del cuerpo y el de los ojos, concluyendo que los más grandes serían más sensibles a la luz que los pequeños (Yack et al., 2004). Con respecto a los microlepidópteros, estudios realizados por de Oliveira et al., (2008) demostraron que los adultos machos y hembras de Tuta absoluta fueron atraídos en forma eficiente por lámparas de luz negra (BLB) y lámparas de UV, las cuales tienen una longitud de onda entre (365 - $400 \mathrm{~nm}$ ).

En cuanto a los diodos emisores de luz (LEDs), éstos están en estado sólido y son fuentes de luz de semiconductores con algunas propiedades específicas, es decir, son pequeños, de alta estabilidad mecánica, alta confiabilidad, largo tiempo de vida operacional, utilizan poca energía y son de bajo costo y ecológicos (Schubert, 2003). Estos LEDs han sido también utilizados en algunas aplicaciones de la vida cotidiana como el control remoto, pantallas numéricas, indicador de estados, pantallas planas, comunicaciones ópticas etc... (Schubert \& Yao, 2002). En general pocos estudios han sido llevados a cabo para evaluar la respuesta de insectos a la luz emitida por LEDs. A su vez, teniendo en cuenta las siguientes características:
1) su monocromía, 2) la amplia variabilidad de colores, 3) el rango de longitud de onda típico se encuentra comprendido entre $20 \mathrm{~nm}$ y $60 \mathrm{~nm}$, con lo cual podemos elegir aquella longitud de onda del color requerido. Por lo anteriormente expuesto, se plantea como objetivo de esta investigación determinar la mejora en la eficiencia de la captura de adultos machos colocando en trampas de agua utilizadas para la captura masiva diodos emisores de luz (LEDs) de diferentes longitudes de onda con la finalidad de poder desarrollar las bases para futuras estrategias integradas a las ya existentes para la prevención y el tratamiento de la plaga.

\section{Objetivo}

Comparar distintos tipos de trampas caseras con luces LEDs en combinación con feromona para la captura de adultos de polilla del tomate (Tuta absoluta).

\section{Materiales y métodos}

\section{Manejo de los invernaderos y del cultivo de tomate}

El ensayo se llevó a cabo en un establecimiento hortícola de producción comercial situado en la localidad de Concordia, localizado en las coordenadas GPS $31^{\circ} 20^{\prime} 28,46^{\prime \prime} \mathrm{S}$; $58^{\circ}$ 2' $0,29^{\prime \prime}$ O; 516 (s.n.m), Departamento de Concordia, Provincia de Entre Ríos, Argentina.

Los muestreos de adultos de polilla del tomate se realizaron en un periodo desde el 15 de agosto de 2016 hasta el 29 de diciembre de 2016. El ensayo se llevó a cabo en dos invernaderos con estructura de madera tipo a Dos Aguas (DA) con una superficie total de $800 \mathrm{~m}^{2}$ cada uno, orientación este-oeste, con las siguientes dimensiones: $16 \mathrm{~m}$ de frente por $50 \mathrm{~m}$ de lateral, con una altura de $2,2 \mathrm{~m}$ en los laterales y $3,5 \mathrm{~m}$ en la parte central con un total de 10 surcos apareados a 1 m entre sí y 0,25 m entre plantas de tomate (c.v., "ELPIDA", Enza Zaden) (redondo híbrido indeterminado), logrando una densidad de plantas de 4 plantas/ $\mathrm{m}^{2}$. Las plantas se condujeron a un sólo tallo y el tutorado se realizó con hilo plástico. En el momento que las plantas alcanzaron la altura de $1,70 \mathrm{~m}$ se bajaron y se apoyó el tallo en el acolchado plástico del surco. El riego y la fertilización se realizaron según las necesidades del cultivo. No se aplicaron hormonas para el cuaje de las flores. 
Los estadios fenológicos del cultivo durante el período que se desarrolló el ensayo fue de crecimiento del fruto, comienzo de maduración, maduración $\mathrm{y}$, finalmente, cosecha. Este establecimiento fue seleccionado por la disposición del productor a que se realice y además por la incidencia de la polilla del tomate en campañas anteriores.

\section{Condiciones climáticas de la zona de muestreo}

En la Figura 2 se observan los valores medios de temperatura y humedad relativa en el período del ensayo.

\section{Tratamientos}

Las trampas que se evaluaron fueron las siguientes:

- Trampa casera con piso engomado y sin feromona (TA) (Figura 3).

- Trampa casera con piso engomado con feromona (TF) (Figura 3).

- Trampa casera con dispositivo LED de $430 \mathrm{~nm}$ superior, piso engomado y sin feromona LBL - F (Figura 4).

- Trampa casera con dispositivo LED de $430 \mathrm{~nm}$ superior, piso engomado y con feromona LBL $+\mathrm{F}$ (Figura 4).

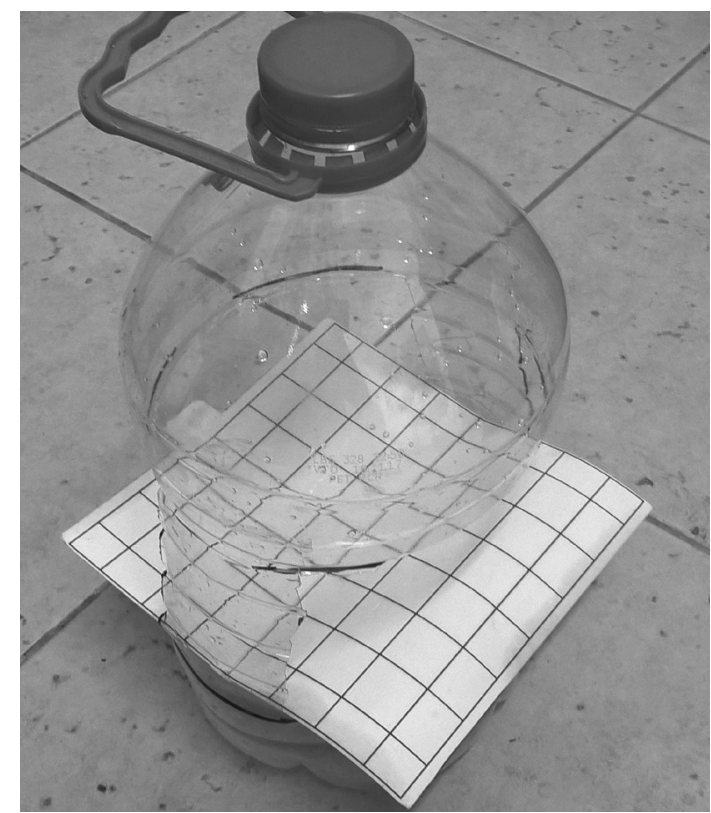

Figura 3. Trampa casera sin luz LED.

- Trampa casera con dispositivo LED de $470 \mathrm{~nm}$ superior, piso engomado y sin feromona LBH - F (Figura 5).

- Trampa casera con dispositivo LED de $470 \mathrm{~nm}$ superior, piso engomado y con feromona LBH $+\mathrm{F}$ (Figura 5).

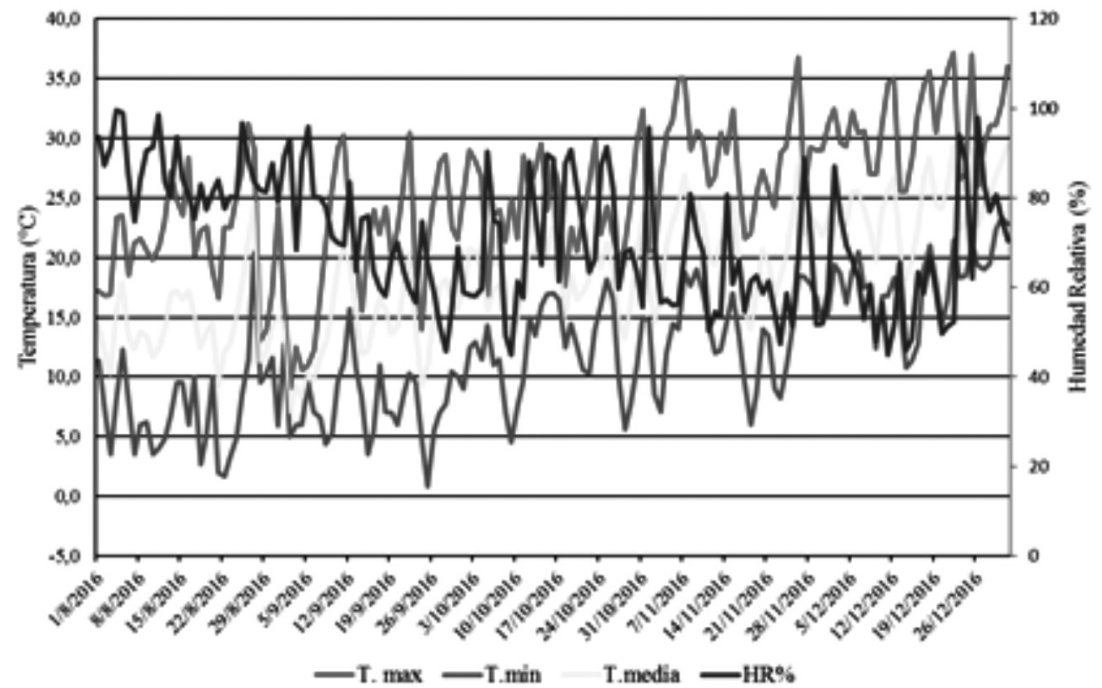

Figura 2. Datos meteorológicos registrados por la estación meteorológica situada en la EEA INTA Concordia durante el periodo del ensayo de agosto de 2016 hasta diciembre del 2016 (valores de temperatura máxima, mínima y media y de humedad relativa). 


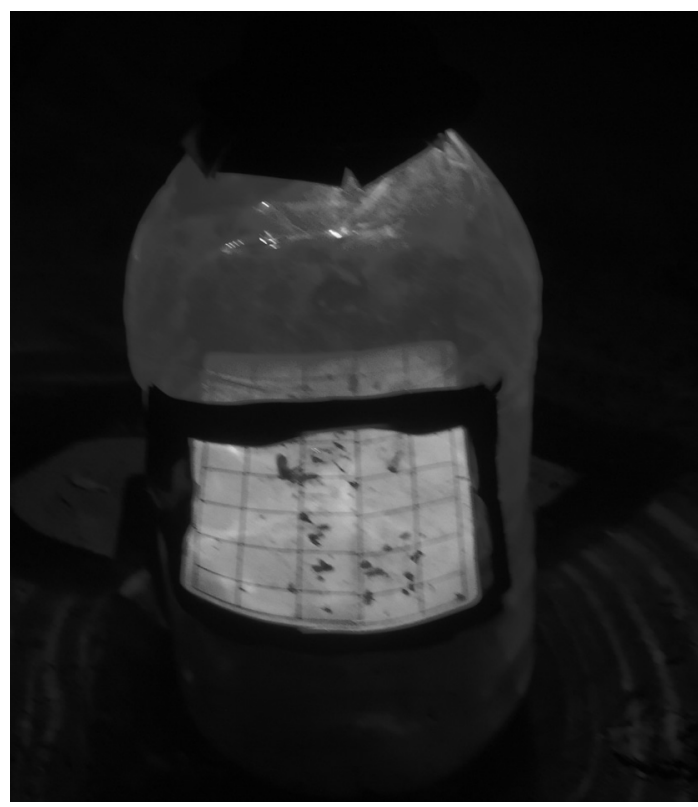

Figura 4. Trampa casera con luz LED $430 \mathrm{~nm}$.

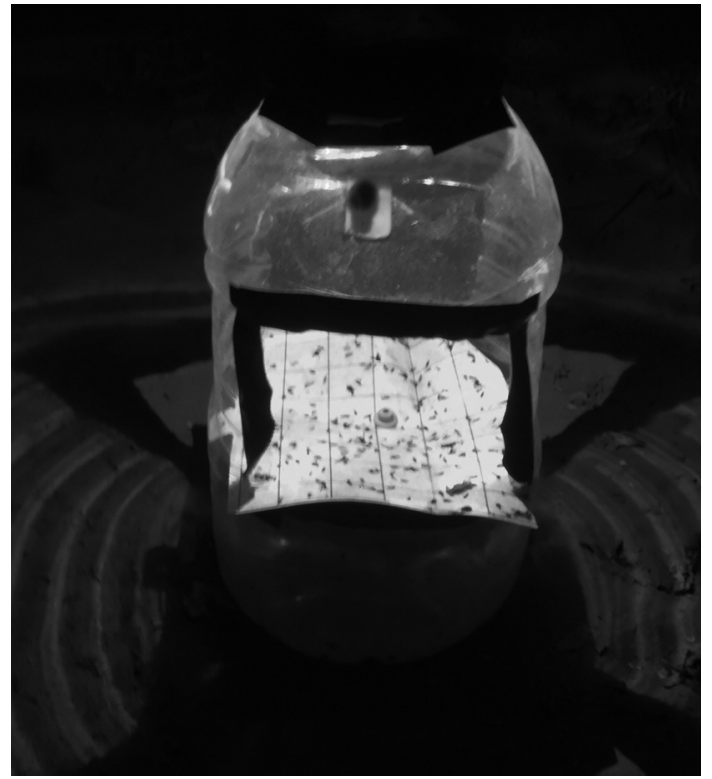

Figura 5. Trampa casera con luz LED $470 \mathrm{~nm}$.

La trampa casera consiste en un bidón de plástico transparente reciclado de 5 litros de capacidad. Para el caso de los tratamientos trampa testigo absoluto sin feromona (TA) y trampa testigo con feromona (TF) en la parte superior del bidón queda cerrado con la misma tapa. Asimismo, en la parte media del bidón se abrieron dos ventanas laterales de $20 \mathrm{~cm}$. de ancho y $20 \mathrm{~cm}$. de alto para permitir el ingreso de los adultos de polilla del tomate y además situar el piso engomado sobre el cual, dependiendo del tratamiento que se utilice, es cebada o no con una feromona como atrayente sexual. Cabe destacar la originalidad de estas trampas por la simplicidad en la confección, su bajo costo y la alimentación a traves de luz solar respecto de las ya conocidas trampas de luz utilizadas para la captura masiva de adultos de la polilla del tomate en invernadero como a campo.

Todas las trampas equipadas con LEDs fueron alimentadas con una fuente de baja tensión proveniente de un panel solar que transforma la energía solar en eléctrica que, a su vez, es acumulada en una batería recargable que está incluida. Asimismo, este dispositivo posee un fotosensor y carga automática (encendido y apagado automático), cuya energía se almacena en una batería AA recargable de 1.2 volts Ni-MH de 2900 mah incluida, lo que permite mantener encendido el LED desde el anochecer hasta el amanecer.

Las trampas LBL- F y LBL + F son trampas TA y TF a las que se les realizó un corte transversal en la parte superior para montar la lámpara LED de 1 Watts de potencia con una longitud de onda de 430nm (SHENZEN SEALAND OPTOELECTRONICS CO., LTD).

Las trampas LBH - F y LBH + F son trampas TA y $\mathrm{TF}$ a las que se les realizó un corte transversal en la parte superior para montar la lámpara LED de 1 Watt de potencia con una longitud de onda de $470 \mathrm{~nm}$ (SHENZEN SEALAND OPTOELECTRONICS CO., LTD).

\section{Diseño del ensayo}

El diseño del ensayo fue en bloques (DBCA) al azar con 5 tratamientos y 2 repeticiones o bloques (invernadero). Las repeticiones estuvieron separadas entre ellas como mínimo 12 metros.

Las trampas se mantuvieron durante aproximadamente 5 meses en invernadero, desde el 1 de agosto al 29 de diciembre de 2016 y fueron colocadas sobre del suelo en los caminos que separan a los camellones para capturar la mayor parte de polillas que emergen del suelo como así también para favorecer la difusión de la feromona y la fuente de luz. Ésta última permaneció encendida desde el anochecer hasta el amanecer y estuvo ubicada por debajo de la altura de los zócalos laterales del invernadero para evitar atraer polillas del exterior. 
Las trampas se rotaron en el invernadero cada 25 días, según indica la Figura 6, de manera que, al cabo de 6 rotaciones, todas las trampas habían pasado por todas las ubicaciones. Esto se realizó de esta manera con el fin de eliminar el efecto de cualquier foco que pudiera existir dentro del invernadero y pudiera influir en las capturas. Asimismo, se decidió cambiar quincenalmente los pisos engomados como así también la feromona sexual de cada una de las trampas en las que fue colocada.

\section{Sistema de evaluación}

Las trampas se colocaron el 15 de agosto de 2016 y se retiraron el 29 de diciembre del mismo año. Para la evaluación de la comparación de las trampas se efectuaron un total de 8 registros quincenales $\mathrm{y}$, posteriormente, se contabilizaron sobre cada una el número total de adultos de polilla del tomate (machos y hembras) que habían quedado adheridas en el piso engomado en laboratorio mediante una lupa estereoscópica de 10 a 40 x de magnificación. Además, semanalmente se monitorearon distintas plagas y enfermedades en las plantas de tomate en ambos invernaderos

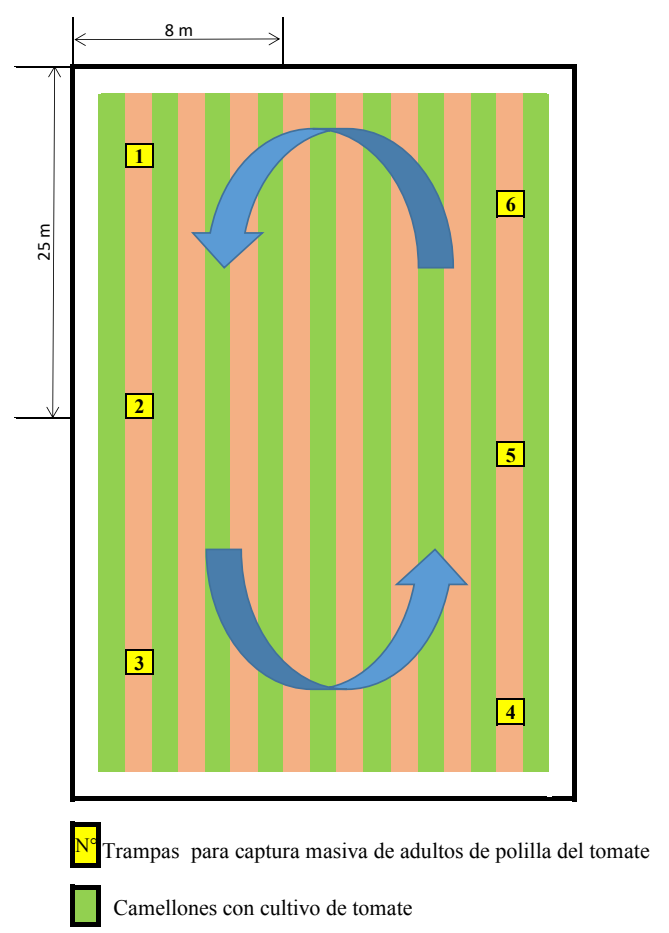

Figura 6. Croquis de la distribución las trampas en invernadero. para observar si alguna de éstas había llegado a un umbral de intervención, así como también para corroborar la carga de las pilas que alimentaban las lámparas LEDs con un multímetro portátil para mantener su funcionamiento desde el amanecer hasta el anochecer.

Los datos del número de capturas de adultos de polillas del tomate que se obtuvieron durante el período del ensayo mostraron un patrón de distribución muy alejado de la distribución Normal con signos de fuerte asimetría. Se observó muy alta frecuencia de valores bajos del número de adultos (0-14 adultos) y poca frecuencia de valores más altos (130-160 adultos). Por esta razón se decidió utilizar un modelo lineal generalizado mixto (Zuur et al., 2009; Di Rienzo et al., 2015), el cual permitió utilizar la distribución Poisson de acuerdo con la naturaleza de la variable respuesta. El modelo contaba con los tratamientos y los invernáculos, como efectos fijos, y las fechas de muestreo, como efecto aleatorio. Las medias de los distintos tratamientos se compararon con la prueba LSD de Fisher con un nivel de significación del 5\%. Se utilizó el paquete estadístico InfoStat.

\section{Resultados}

\section{Registros de capturas de la polilla del tomate}

Los resultados estadísticos obtenidos durante el período del ensayo permitieron detectar diferencias significativas del número de adultos de polilla del tomate capturados por tipo de trampa $\left(\mathrm{X}_{\text {tratamiento }}^{2}=3135,86 ; \mathrm{df}=5\right.$ : $\left.\mathrm{p}<0.0001\right)$.

En la Tabla 1 y Figura 7 se observan dos grupos de significación bien diferenciados, los constituidos por los tipos de trampa que combinan lámparas LEDs con feromona sexual y el otro grupo con los tipos de trampas con LEDs y feromona sin combinar, siendo las trampas del primer grupo las que mayor capturas promedio registraron (76,68 adultos de polilla del tomate/LBH+F) y (70,37 adultos de polilla del tomate/LBL+F). En el caso de las trampas no combinadas, la trampa equipada con feromona fue la que obtuvo mayores capturas (34,62 adultos de polilla del tomate/TF) y le seguían por un amplio margen las trampas con fuente de luz (5.75 polillas del tomate/ LBHF), (4.56 polilla del tomate/LBL-F 4.56) y, por último, la trampa sin feromona (2.06 polillas del tomate/ TA). 
Tabla 1. Resultados de test de comparación de medias ajustadas del número de capturas de Tuta absoluta (media \pm Error Standard) para cada uno de los tratamientos evaluados.

\begin{tabular}{lc}
\hline \multicolumn{1}{c}{ Tipo de trampa } & (Medias \pm D.S.) \\
\hline Trampa casera en bidón con LED 470 nm con feromona (LBH+F) & $35.16 \pm 22.59 \mathrm{a}$ \\
Trampa casera en bidón con LED 430 nm con feromona (LBL+F) & $33.60 \pm 21.59 \mathrm{a}$ \\
Trampa casera en bidón con feromona (TF) & $17.07 \pm 10.98 \mathrm{~b}$ \\
Trampa casera en bidón con LED 470 nm sin feromona (LBH-F) & $2.64 \pm 1.71 \mathrm{c}$ \\
Trampa casera en bidón con LED 430 nm sin feromona (LBL-F) & $2.09 \pm 1.36 \mathrm{c}$ \\
Trampa casera en bidón sin feromona (TA) & $1.02 \pm 0.68 \mathrm{~d}$ \\
\hline
\end{tabular}

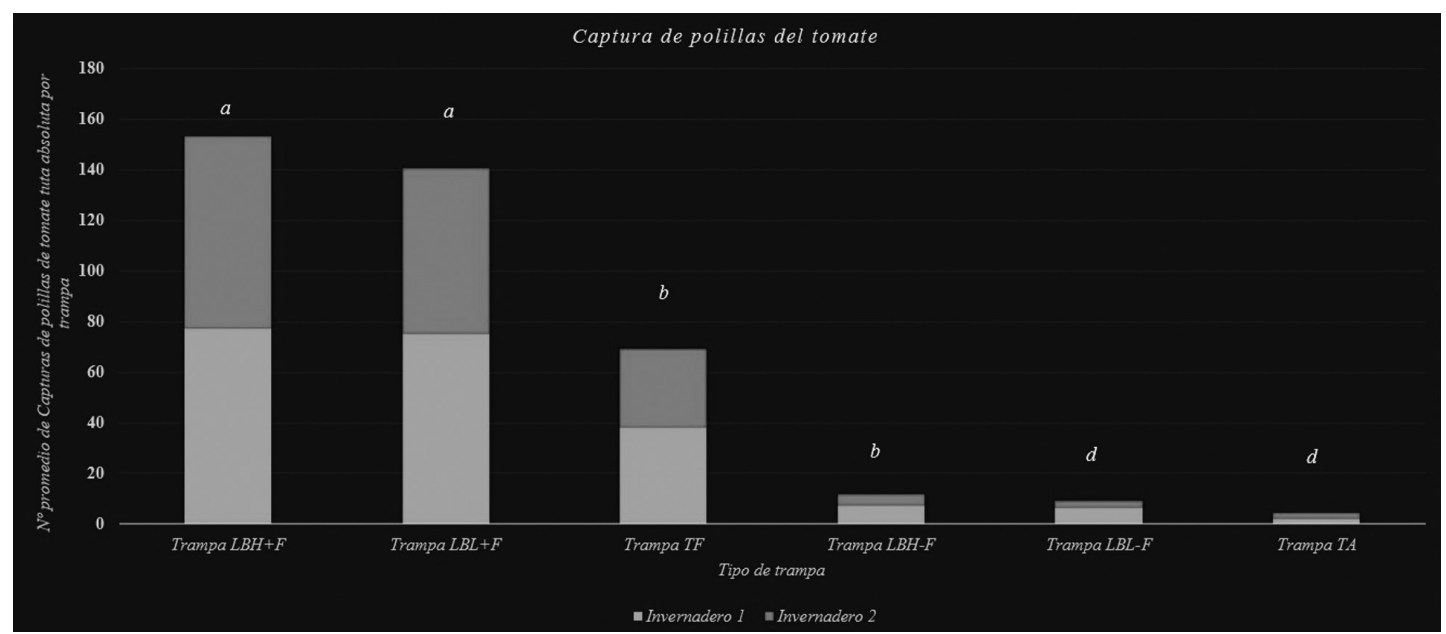

Figura 7. Número promedio de polillas del tomate por trampa y por tipo de trampa durante el periodo del ensayo.

\section{Discusión y conclusión}

Los datos presentados en la Tabla 1 muestran que la T. absoluta es atraída por fuente de luz de longitudes de onda de $430 \mathrm{~nm}$ y $470 \mathrm{~nm}$ como señal de orientación luego de observar un mayor número de capturas en trampas sólo cuando éstas estaban en combinación con atrayentes, en este caso feromona sexual. Estos datos son coincidentes con los obtenidos por (Matos et al., 2012), quienes evaluaron diferentes tipos de trampas para captura masiva de adultos de T. absoluta tomate y registraron mayor captura en las que se combinaba una señal química, en este caso feromona sexual, con un dispositivo lumínico. Asimismo, es conocido que en el orden Lepidoptera en general tienen receptores con sensibilidad espectral en la región del azul con picos cercanos a los 460nm (Briscoe \& Chittka, 2001). Dentro de los lepidópteros, la polilla del tabaco (Manduca sexta) ha sido durante mucho tiempo un insecto experimental utilizado para diferentes estudios referidos a los potenciales eléctricos en respuesta al estímulo lumínico, que permitieron conocer que su ojo contiene tres diferentes tipos de fotorreceptores con sensibilidad espectral, en el pico del espectro UV (370 nm - $390 \mathrm{~nm})$, azul $(450$ - $470 \mathrm{~nm})$ y la región verde del espectro (370 a $(530-550 \mathrm{~nm})$, provocando así la estimulación de cada uno de los fotorreceptores a diferentes respuestas en su comportamiento (Cutler et al., 1995). Sobre la base de los resultados y las citas anteriormente mencionadas, podemos concluir que las trampas de feromonas en combinación con los dispositivos LEDs de longitudes de onda de $430 \mathrm{~nm}$ y $470 \mathrm{~nm}$ produjeron un sinergismo que funcionó como orientación o señal de navegación que dio como resultado el máximo de las capturas en ambos invernaderos.

De los 6 tipos de trampas evaluadas, las que tenían montadas luces LEDs $(\mathrm{LBH}+\mathrm{F}$ y $\mathrm{LBL}+\mathrm{F}$ ) fueron las que obtuvieron un número significativamente mayor de capturas de polilla del tomate, seguida por la trampa con feromona 
(TF) la cual registró una captura de polillas significativamente menor con respecto a las anteriores pero mayor al resto de las trampas (LBHF, LBL-F, TA). Éstas últimas no contabilizaron caídas de adultos de polilla significativamente diferentes entre sí.

En la comparación entre los tipos de trampas con LEDs $(\mathrm{LBH}+\mathrm{F}$ y $\mathrm{LBL}+\mathrm{F})$ no se obtuvieron diferencias significativas. A continuación, se exponen las ventajas y desventajas de las trampas LEDs:

\section{Ventajas:}

1) Su bajo costo al igual que el de los paneles solares para el armado del dispositivo de captura masiva;

2) Alimentación autónoma, lo cual no exige ningún tipo de alimentación de red eléctrica y además brinda mayor seguridad en su manipulación por su bajo voltaje.

3) Su bajo mantenimiento y la adaptabilidad de su funcionamiento a diferentes condiciones meteorológicas, incluso a campo bajo la lluvia.

\section{Desventajas:}

1) Su colocación debe realizarse de manera tal que el cultivo no obstaculice la emisión de luz ni en los lugares sin sombra para permitir la carga completa de la batería.

2) Su ubicación en lugares donde no emita señal de llamada a insectos externos al invernadero, en nuestro caso particular, en los cuales los invernaderos no estén cerrados herméticamente por mallas por debajo de la altura del zócalo.
Tomando en consideración que es una técnica no contaminante para el medio ambiente y que su utilización adecuada puede ser una valiosa herramienta como complemento de otras que constituyen el manejo integrado de plagas (MIP), esto plantea la necesidad de seguir investigando este tipo de trampas con la finalidad de aumentar su eficacia para realizar la captura de polilla de tomate.

\section{Agradecimientos}

Este trabajo pudo ser realizado gracias al apoyo de:

1) Proyecto específico INTA - PNHFA 1106082. "Tecnología apropiada para la sustentabilidad con énfasis en sistemas hortiflorícolas con énfasis en cultivos protegidos".

2) Red de Agroecologia INTA - REDAE 1136021

3) Proyecto Regional con Enfoque Territorial INTA - PRETERIOS 1263305." Contribuir al desarrollo socio económico del noreste de Entre Ríos, en un marco de competitividad, salud ambiental y equidad social".

5) A la familia del productor Víctor Campeglia, que siempre me recibió con buena disposición, durante los muestreos.

4) A la traductora Pública en Inglés Mónica Castresana, por sus aportes, observaciones y correcciones.

6) A los Ingenieros en electrónica Gonzalo Castresana y Jorge Jones, por sus aportes en la parte electrónica.

7) Al Ing. Agr. Javier Rosenbaum por el uso del vehículo asignado a PROHUERTA en los momentos en que no había disponibilidad de otros.

\section{Literatura Citada}

Al-Zaidi, S.

2009. Recommendations for the Detection and Monitoring of Tuta absoluta. Russell IPM. Disponible en http://www. russellipm-agriculture.com/case-studies/tuta-absoluta/ Consultado: 19/ene/2017.

Bogorni, P.C.; da Silva, R.A. \&G.S. Carvalho.

2003. Consumo de mesofilo foliar por Tuta absoluta (Meyrick, 1971) (Lepidoptera: Gelechidae) em tres cultivares de Lycopersicon esculentum Mill. Ciencia Rural, 33 (1): 7-11.

Briscoe, A.D., \& L. Chittka.

2001. The evolution of color vision in insects. Annual Review of Entomology, 46: 471-510.
Cabello, T., Gallego, J.R., Fernández, F.J., Vila, E., Soler, A. \& A. Parra.

2010. Aplicación de parasitoides de huevos en el control de Tuta absoluta en España. Phytoma España, 217: 53-59.

Castelo-Branco, M.

1992. Flutuação populacional da traça-do-tomateiro na região do Distrito Federal. Horticultura Brasileira, 10: 33-34.

Cutler, D.E., Bennett, R.R., Stevenson, R.D \& R.H. White. 1995. Feeding-behavior in the nocturnal moth Manduca sexta is mediated mainly by blue receptors, but where are they located in the retina. Journal of Experimental Biology, 198: 1909-1917. 
Desneux, N., Wajnberg, E., Wyckhuys, K.A.G., Burgio, G. \& S. Arpaia.

2010. Biological invasion of European tomato crops by Tuta absoluta: ecology, geographic expansion and prospects for biological control. Journal of Pest Science, 83:197-215.

Di Rienzo J.A., Casanoves F., Balzarini M.G.; Gonzalez L.;

Tablada M., Robledo C.W.

2015. Grupo InfoStat, FCA, Universidad Nacional de Córdoba, Argentina.

de Oliveira, A.C.R., Veloso, V.R.S., Barros, R.G., Fernandes,

P.M. \& E.R.B., de Souza.

2008. Captura de Tuta absoluta (Meyrick) (Lepidoptera: Gelechiidae) com armadilha luminosa na cultura do tomateiro tutorado. Pesquisa Agropecuária Tropical, 38: 153-157. EPPO.

2011. Tuta absoluta continues to spread around the Mediterranean Basin (2011/076). EPPO Reporting Services 4 (076). 30 p.

Ferrara, F.A., Vilela, E.F., Jham, G.N., Eiras, A.E., Picanço, M.C., Attygalle, A.B., Svatos, A., Frighetto, R.T.S. \& J. Meinwald.

2001. Evaluation of the synthetic major component of the sex pheromone of Tuta absoluta (Myerick) (Lepidoptera: Gelechiidae). Journal of Chemical Ecology, 27 (5): 907-917.

Frank, K.D.

2006. Effects of artificial night lighting on moths. In: Rich, C., Longcore, T. (Eds.), Ecological Consequences of Artificial Night Lighting. Island Press. Washington DC, Us. pp. 305-344.

Giordano, L., Silva, C.

1999. Hibridação em tomate. In: BORÉM, A. (Ed.). Hibridação artificial de plantas. UFV. Viçosa, Brasil. pp. 463-480.

Harizanova, V., Stoeva A., Mohamedova M.

2009. "Tomato Leaf Miner, Tuta absoluta (Povolny) (Lepidoptera: Gelechiidae) - First Record in Bulgaria", Agricultural Science and Technology, 1 (3): 95-98.

Leite, G.L.D., Picanço, M., Jham, G.N., \& Marquini, F. 2004. Intensity of Tuta absoluta (Meyrick, 1917) (Lepidoptera: Gelechiidae) and Liriomyza spp. (Diptera: Agromyzidae) attacks on Lycopersicum esculentum mill. Leaves. Ciência e Agrotecnologia, 28: 42-48.
Martí Martí S., Muñoz Celdrán, Casagrande E.

2010. El uso de feromonas para el control de Tuta absoluta: primeras experiencias en campo. Phytoma España, 217: 35-40.

Matos, T., Figueiredo, E. \& A. Mexia.

2012. Sexual pheromone traps with light for mass trapping of Tuta Absoluta (Meyrick), yes ou no?. Revista de Ciências Agrárias, 35, (2): 282-286.

Picanço, M.C., Bacci, L., Crespo A.L., Miranda M.M. \& J.C. Martins.

2007. Effect of integrated pest management practices on tomato production and conservation of natural enemies. Agricultural and Forest Entomology, 9, (4): 327-335.

Potting, R.; van der Gaag, D.J.; Loomans, A.; van der Straten, M.; Anderson, H.; MacLeod, A.; Guitián Castrillón, J.M.; Cambra, G.V.

2013. Tuta absoluta, tomato leaf miner moth or South American tomato moth, Ministry of Agriculture, Nature and Food Quality, Plant Protection Service of the The Netherland.Wageningen,The Netherlands. 28 pp.

Schubert, E.F.

2003. Light-Emitting Diodes. Cambridge University Press, Cambridge, New York. 313 p.

Siqueira, H.A.A., Guedes, R.N.C. \& M.C. Picanço.

2000. Cartap resistance and synergism in populations of Tuta absoluta (Lep., Gelechiidae). Journal Applied Entomology, 124: 233-238.

Van Deventer, P.

2009. Leaf miner threatens tomato growing in Europe. Fruit and Vegetal Technology, 9: 10-12.

Yack, J.E., Johnson, S.E., Brown, S.G. \& E.J. Warrant.

2007. The eyes of Macrosoma sp. (Lepidoptera: Hedyloidea): A nocturnal butterfly with superposition optics. Arthropod Structure \& Development, 36; 11-22.

Zuur, A.F.; Ieno, E.N.; Walker, N.J.; Saveliev, A.A. y Smith, G.M. 2009. Zero-truncated and zero-inflated models for count data. Mixed effects models and extensions in ecology vith R. ED Springer New York, US. pp. 261-293. 
